BMJ Open Diabetes

Research \& Care

\title{
Relationships of surrogate indexes of insulin resistance with insulin sensitivity assessed by euglycemic hyperinsulinemic clamp and subclinical vascular damage
}

\author{
Teresa Vanessa Fiorentino (D) , ${ }^{1}$ Maria Adelaide Marini, ${ }^{2}$ Elena Succurro, ${ }^{1}$ \\ Francesco Andreozzi, ${ }^{1}$ Giorgio Sesti ${ }^{3}$
}

To cite: Fiorentino TV, Marini MA, Succurro E, et al. Relationships of surrogate indexes of insulin resistance with insulin sensitivity assessed by euglycemic hyperinsulinemic clamp and subclinical vascular damage. BMJ Open Diab Res Care 2019;7:e000911. doi:10.1136/ bmjdrc-2019-000911

- Additional material is published online only. To view please visit the journal online (http://dx.doi.org/10.1136/ bmjdrc-2019-000911).

Received 14 September 2019 Revised 8 0ctober 2019 Accepted 16 October 2019

Check for updates

(C) Author(s) (or their employer(s)) 2019. Re-use permitted under CC BY-NC. No commercial re-use. See rights and permissions. Published by BMJ.

${ }^{1}$ Department of Medical and Surgical Sciences, Magna Graecia University of Catanzaro, Catanzaro, Italy

2Department of Systems Medicine, University of Rome Tor Vergata, Roma, Lazio, Italy ${ }^{3}$ Department of Clinical and Molecular Medicine, University of Rome La Sapienza, Roma, Lazio, Italy

Correspondence to Dr Teresa Vanessa Fiorentino; vanessa.fiorentino@unicz.it

\section{ABSTRACT}

Introduction Insulin resistance plays a crucial role in the pathogenesis of type 2 diabetes and cardiovascular disease. The triglyceride to high-density lipoprotein cholesterol (TG/HDL-C) ratio, visceral adiposity index (VAl), lipid accumulation product (LAP) and triglycerides $\times$ fasting glucose (TyG) index are surrogate measures of insulin sensitivity based on anthropometric and/or biochemical parameters routinely collected in clinical practice. Herein, we compared the relationships of these four surrogate indexes with insulin sensitivity assessed by the gold standard euglycemic hyperinsulinemic clamp technique, and subclinical vascular damage.

Research design and methods 631 subjects with different degrees of glucose tolerance underwent euglycemic hyperinsulinemic clamp. The surrogate TG/ HDL-C ratio, VAl, LAP and TyG indexes were computed. Pulse pressure and carotid intima-media thickness (IMT) were measured as indicators of subclinical vascular damage.

Results All the four surrogate indexes showed a significant correlation with insulin-stimulated glucose disposal in the whole study population. However, only LAP index had a significant association with insulin sensitivity across the different glucose tolerance groups. LAP index showed the highest area under the receiver operating characteristic curve $(0.728)$ to detect individuals with insulin resistance defined as the bottom quartile of insulin-stimulated glucose disposal, followed by TG/HDL-C ratio (0.693), TyG index (0.688) and VAl (0.688). A significant association was found between the four indexes of insulin sensitivity and pulse pressure and IMT. All the four indexes have a similar ability to detect individuals with vascular atherosclerosis defined by IMT $>0.9 \mathrm{~mm}$. Conversely, LAP index had the greatest ability to recognize individuals with increased vascular stiffness defined by pulse pressure $\geq 60 \mathrm{~mm} \mathrm{Hg}$.

Conclusion Among the surrogate TG/HDL-C ratio, VAl, LAP and TyG indexes of insulin sensitivity, LAP index showed a significant association with insulin-stimulated glucose disposal across the different glucose tolerance categories and the highest ability to detect insulin resistance and subclinical vascular damage.

\section{Significance of this study}

What is already known about this subject?

- The triglyceride to high-density lipoprotein cholesterol (TG/HDL-C) ratio, visceral adiposity index (VAl), lipid accumulation product (LAP) and triglycerides $\times$ fasting glucose (TyG) index have been developed as surrogate measures of insulin resistance based on anthropometric and/or biochemical parameters routinely collected in clinical practice.

What are the new findings?

$>$ Among the four surrogate indexes of insulin resistance, LAP index correlates better with insulin sensitivity assessed by the gold standard euglycemic hyperinsulinemic clamp technique.

- LAP index was the only surrogate index showing significant association with the insulin sensitivity in individuals with impaired glucose tolerance or type 2 diabetes.

- The ability of LAP index to identify subjects with insulin resistance is higher than TG/HDL-C ratio, VAl and TyG indexes.

- All the four indexes have a similar ability to detect individuals with subclinical atherosclerosis. Conversely, LAP index had the greatest ability to recognize individuals with increased vascular stiffness.

How might these results change the focus of research or clinical practice?

- Among the four surrogate measures of insulin resistance based on anthropometric and biochemical parameters, LAP index is the best discriminator of insulin resistance and subclinical vascular damage.

- Assessment of LAP index in clinical practice should be preferred to TG/HDL-C ratio, VAl and TyG indexes for a better cardiometabolic risk stratification.

\section{INTRODUCTION}

Insulin resistance is a characteristic trait of both pre-diabetes $^{1-3}$ and metabolic syndrome. ${ }^{4-6}$ Insulin resistance is considered central to the pathophysiology of type 2 diabetes, ${ }^{7}$ and predicts the development of cardiovascular 
disease. $^{9-11}$ Although insulin resistance is an important determinant of cardiometabolic diseases, a direct measure of insulin sensitivity by the gold standard euglycemic hyperinsulinemic clamp technique ${ }^{12}$ is impractical for large-scale epidemiological studies, due to the laborious, expensive, and time-consuming procedure required. Therefore, many surrogate indexes of insulin sensitivity have been proposed using anthropometric variables, fasting insulin and/or glucose levels or insulin and glucose levels during an oral glucose tolerance test (OGTT) ${ }^{13-15}$ An ideal surrogate index of insulin sensitivity for use in large epidemiological studies should be based on affordable anthropometric and/or laboratory measures routinely gathered in clinical settings. Among these, the visceral adiposity index (VAI) ${ }^{16}$ and the lipid accumulation product (LAP), both based on anthropometric and laboratory parameters ${ }^{17}$ have been developed and analyzed in relation to homeostatic model assessment of insulin resistance (HOMA-IR), but not to the gold standard measure of insulin sensitivity assessed by the euglycemic hyperinsulinemic clamp method. ${ }^{18-25}$ Additionally, two other surrogate indexes of insulin sensitivity based on plasma triglyceride (TG) and high-density lipoprotein cholesterol (HDL-C) measurements, that is, the triglycerides $\times$ fasting glucose $(\mathrm{TyG})$ index and TG/HDL-C concentration ratio, have been suggested as surrogate estimates of insulin sensitivity, and validated in comparison to the euglycemic hyperinsulinemic clamp method, ${ }^{26}$ the steady-state plasma glucose (SSPG) concentration during the insulin suppression test ${ }^{27}$ or a 3-hour hyperglycemic clamp test. ${ }^{28}$ Although these two lipid-based surrogate indexes have been studied in non-diabetic individuals, in subjects with impaired fasting glucose (IFG), and in patients with type 2 diabetes, ${ }^{26-28}$ it is unknown whether they might be useful in the identification of insulin resistance in individuals with impaired glucose tolerance (IGT). In the present study, we pursued two aims: (1) to compare VAI, LAP, TyG, and TG/HDL-C ratio surrogate indexes with the euglycemic hyperinsulinemic clamp method, the gold standard measure of insulin sensitivity, in a large cohort of European individuals with different degrees of glucose tolerance, and (2) to examine the relationship of surrogate measures of insulin sensitivity with classical and inflammatory cardiovascular risk factors, as well as carotid intima-media thickness (IMT), a reliable index of subclinical atherosclerosis ${ }^{29}$ related to insulin resistance. ${ }^{30}$

\section{METHODS}

\section{Study subjects}

The study sample includes 631 adults (age $>18$ years) consecutively recruited at the Department of Systems Medicine, University of Tor Vergata, Rome, and at the Department of Medical and Surgical Sciences of the University 'Magna Graecia' of Catanzaro, Italy, according to a previously described protocol. ${ }^{331}$ On the first day, after an overnight fasting, all subjects underwent anthropometrical evaluation including measurements of body mass index (BMI), waist circumference, and body composition evaluated by bioelectrical impedance. Height was measured to the nearest $0.1 \mathrm{~cm}$, while body weight was measured with a calibrated electronic scale to the nearest $0.1 \mathrm{~kg}$. Waist circumference was measured at the umbilical level, at the midpoint between the iliac crest and the rib cage. The BMI was calculated as body weight in kilograms divided by height in square meters $\left(\mathrm{kg} / \mathrm{m}^{2}\right)$. Blood pressure was measured after a 5 min rest at the right upper arm, in the sitting position, with a sphygmomanometer for three times and the mean was used in the analyses. Pulse pressure was calculated as the difference between systolic blood pressure and diastolic blood pressure. A value of pulse pressure $\geq 60 \mathrm{~mm} \mathrm{Hg}$ was used as index of increased vascular stiffness. ${ }^{32}$ A $75 \mathrm{~g}$ OGTT was performed with basal and 2-hour postload sampling for plasma glucose assays. On the second day, after a 12-hour fasting, participants underwent a euglycemic hyperinsulinemic clamp study, as previously described. ${ }^{33}$ Briefly, a priming dose of insulin was administrated during the early $10 \mathrm{~min}$ to acutely increase plasma insulin followed by continuous insulin infusion fixed at $40 \mathrm{mU} / \mathrm{m}^{2} \times \min$. Blood glucose levels were maintained constant during the 2-hour clamp study by infusing $20 \%$ glucose at varying rates according to blood glucose measurements assessed by a glucose analyzer at 5 min intervals. Glucose metabolized by the whole body (M) was calculated as the mean rate of glucose infusion measured during the last $60 \mathrm{~min}$ of the clamp examination (steady state) and was expressed as milligrams per minute per kilogram fat-free mass $\left(\mathrm{M}_{\mathrm{FFM}}\right)$. Subjects were considered insulin resistant when they were in the lower quartile of $\mathrm{M}_{\mathrm{FFM}}$ values of the study cohort.

Carotid IMT was measured by ATL HDI 3000 ultrasound system (Advanced Technology Laboratories, Bothell, WA) equipped with a $5 \mathrm{MHz}$ linear array transducer as previously described. ${ }^{34}$ For each patient two measurements were performed bilaterally, and the values were averaged, to obtain the mean of IMT of the common carotid artery. Ultrasound examinations were performed by a skilled examiner who was unaware of clinical and laboratory findings of participants. A value of IMT $>0.9 \mathrm{~mm}$ was used as index of vascular atherosclerosis. ${ }^{32}$

According to the American Diabetes Association (ADA) criteria, ${ }^{35}$ individuals were classified as having normal glucose tolerance (NGT) when fasting glucose levels were $<100 \mathrm{mg} / \mathrm{dL}(5.5 \mathrm{mmol} / \mathrm{L})$ and 2-hour postload $<140 \mathrm{mg} /$ $\mathrm{dL}(<7.77 \mathrm{mmol} / \mathrm{L})$, isolated IFG when fasting glucose levels were $100-125 \mathrm{mg} / \mathrm{dL}(5.5-6.9 \mathrm{mmol} / \mathrm{L})$ and 2-hour postload $<140 \mathrm{mg} / \mathrm{dL}(<7.77 \mathrm{mmol} / \mathrm{L})$, IGT when fasting glucose levels were $<126 \mathrm{mg} / \mathrm{dL}(<7 \mathrm{mmol} / \mathrm{L})$ and 2-hour postload was $140-199 \mathrm{mg} / \mathrm{dL}(7.77-11.0 \mathrm{mmol} / \mathrm{L})$, and newly diagnosed type 2 diabetes when fasting glucose levels were $\geq 126 \mathrm{mg} / \mathrm{dL}(\geq 7 \mathrm{mmol} / \mathrm{L}$ ) or 2-hour postload was $\geq 200 \mathrm{mg} / \mathrm{dL}(\geq 11.1 \mathrm{mmol} / \mathrm{L})$.

\section{Analytical determinations}

Glucose, TGs, total cholesterol and HDL-C concentrations were determined by enzymatic methods (Roche, Basel, Switzerland). High-sensitivity C-reactive protein (hsCRP) levels were measured by automated instrument 
BN II System analyzer (Siemens Healthcare, Marburg, Germany) with the CardioPhase hsCRP kit. Uric acid concentrations were determined by the uricase/peroxidase method implemented in an autoanalyzer (Boehringer Mannheim, Mannheim, Germany). Plasma insulin concentrations were measured by a chemiluminescencebased assay (Immulite, Siemens, Italy).

\section{Definition of LAP, VAI, TyG, and TG/HDL-C ratio surrogate indexes}

The LAP index was calculated as (waist circumference $65) \times($ TGs $[\mathrm{mmol} / \mathrm{L}])$ in men, and (waist circumference $-58) \times($ TG $[\mathrm{mmol} / \mathrm{L}])$ in women. ${ }^{17}$ The VAI was index calculated using gender-specific formulas: men (waist circumference / $39.68+[1.88 \times \mathrm{BMI}]) \times($ TGs $/ 1.03) \times$ (1.31/HDL-C); women: (waist circumference / $36.58+$ $[1.89 \times \mathrm{BMI}]) \times(\mathrm{TGs} / 0.81) \times(1.52 / \mathrm{HDL}-\mathrm{C})$, where both TG and HDL-C levels are expressed in $\mathrm{mmol} / \mathrm{L}{ }^{16}$ The TyG index was calculated as the $\mathrm{Ln}$ [fasting TGs $(\mathrm{mg} / \mathrm{dL})$ $\times$ fasting glucose $(\mathrm{mg} / \mathrm{dL}) / 2] .{ }^{26}$ The TG/HDL-C ratio was calculated as the ratio of TG $(\mathrm{mg} / \mathrm{dL})$ to HDL-C $(\mathrm{mg} /$ dL). ${ }^{27}$

\section{Statistical analysis}

TG/HDL-C ratio, LAP and VAI indexes, hsCRP, and TG values were natural $\log$ transformed for statistical analysis due to their skewed distribution. Continuous data are expressed as means \pm SD. A general linear model with adjustment for age and gender was used to compare anthropometric and metabolic differences between the groups. Categorical variables were compared by $\chi^{2}$ test. Relationships between variables were determined by Pearson's correlation coefficient ( $r$ ). As a measure of discrimination, the ability of each index to detect subjects with insulin resistance, defined as the lower quartile of $\mathrm{M}_{\mathrm{FFM}}$ values, vascular atherosclerosis, defined as IMT $>0.9 \mathrm{~mm}$, and increased vascular stiffness, defined as a pulse pressure $\geq 60 \mathrm{~mm} \mathrm{Hg},{ }^{32}$ was assessed by the area under the receiver operating characteristic (ROC) curve. The area under the ROC curve (AUC) was used as a measure of how well surrogate indexes of insulin sensitivity identify insulin-resistant individuals, vascular damage or increased vascular stiffness. An AUC of 1.0 points to perfect classification of individuals with insulin resistance or organ damage, whereas 0.5 means that the classification is not better than chance. Statistical differences between the AUCs were determined by the method described by DeLong et al. ${ }^{36}$ Youden index was used to derive the optimal threshold of each marker to detect insulin resistance, vascular damage, and increased vascular stiffness. All tests were two sided, and a $p$ value $<0.05$ was considered statistically significant. All calculations were performed with SPSS V.22.0 software program for Windows.

\section{RESULTS}

A total of 631 individuals ( 350 women and 281 men) were enrolled, including 446 subjects with NGT, 60 subjects with
IFG, 95 subjects with IGT, and 30 individuals with newly diagnosed type 2 diabetes. In the whole study sample, the mean age was $39.6 \pm 10.7$ years, BMI was $30.0 \pm 7.1 \mathrm{~kg} / \mathrm{m}^{2}$, and the proportion of participants with family history of type 2 diabetes was $75 \%$. Anthropometric and biochemical features of the study participants grouped on the basis of glucose tolerance are shown in table 1. Deterioration of glucose homeostasis from NGT to type 2 diabetes was associated with age and several measures of adiposity, cardiometabolic and subclinical atherosclerosis including BMI, visceral adiposity, systolic blood pressure, pulse pressure, HDL, TGs, fasting and 2-hour postload glucose, uric acid, hsCRP, and IMT. As expected, deterioration of glucose homeostasis was associated with a graded decline in insulin sensitivity directly measured as insulin-stimulated glucose disposal $\left(\mathrm{M}_{\mathrm{FFM}}\right)$ by euglycemic hyperinsulinemic clamp study, and a graded increase in the values of the surrogate indexes of insulin resistance LAP, VAI, TyG, and TG/HDL-C concentration ratio (table 1).

Correlations of surrogate indexes of insulin resistance with insulin sensitivity assessed by euglycemic hyperinsulinemic clamp study

Table 2 shows the Pearson's correlation coefficient between surrogate indexes of insulin resistance with insulin sensitivity directly assessed by euglycemic hyperinsulinemic clamp. All the four indexes showed a significant correlation with insulin-stimulated glucose disposal $\left(\mathrm{M}_{\mathrm{FFM}}\right)$ in the whole study group. However, when the relationships were examined across glucose tolerance groups the correlations remained statistically significant in subjects with NGT and IFG, but not among those with IGT or type 2 diabetes with the exception of LAP index (table 2). Indeed, the LAP index exhibited the stronger correlation with insulin-stimulated glucose disposal $\left(\mathrm{M}_{\mathrm{FFM}}\right)$ than did the other indexes across glucose tolerance groups. A significant difference in correlation coefficients using the Steiger's $\mathrm{Z}$ test was observed by comparing LAP index and VAI $(\mathrm{p}=0.04)$ or TyG $(\mathrm{p}=0.03)$, but not in comparison with TG/ HDL-C ratio $(\mathrm{p}=0.10)$.

As a measure of discrimination, the ability of surrogate indexes to detect individuals with insulin resistance defined as the bottom quartile of $\mathrm{M}_{\mathrm{FFM}}$ values was assessed by the AUC. As shown in table 3, and online supplementary figure 1, LAP index had the highest AUC followed by TG/HDL-C ratio, TyG index and VAI in the whole study group.

\section{Correlations of surrogate indexes of insulin resistance with} cardiometabolic risk factors and subclinical vascular damage and stiffness

Table 4 shows the Pearson's correlation coefficient between surrogate indexes of insulin resistance with cardiometabolic risk factors and indicators of vascular damage including pulse pressure and IMT. All the four indexes showed a highly significant correlation with systolic and diastolic blood pressure, pulse pressure, uric acid levels, hsCRP, and IMT.

We evaluated the ability of surrogate indexes of insulin resistance to detect individuals with 
Table 1 Clinical characteristics of study population subdivided according to glucose tolerance status

\begin{tabular}{lllllll}
\hline & $\begin{array}{l}\text { Whole } \\
\text { sample }\end{array}$ & NGT & IFG & IGT & $\begin{array}{l}\text { Type } 2 \\
\text { diabetes }\end{array}$ & P value \\
\hline Gender (male/female) & $281 / 350$ & $179 / 267$ & $41 / 19$ & $42 / 53$ & $19 / 11$ & $<0.0001$ \\
\hline Age (years) & $39.6 \pm 10.7$ & $37.4 \pm 10.0$ & $43.4 \pm 10.1$ & $44.3 \pm 10.4$ & $50.6 \pm 10.3$ & $<0.0001$ \\
\hline BMI (kg/m ) & $30.0 \pm 7.1$ & $29.3 \pm 7.2$ & $29.8 \pm 5.8$ & $33.2 \pm 6.6$ & $31.7 \pm 7.4$ & $<0.0001^{*}$ \\
\hline Waist circumference (cm) & $97 \pm 15$ & $94 \pm 15$ & $97 \pm 13$ & $104 \pm 13$ & $106 \pm 17$ & $<0.0001^{*}$ \\
\hline Systolic blood pressure (mm Hg) & $126 \pm 16$ & $123 \pm 16$ & $132 \pm 14$ & $133 \pm 17$ & $138 \pm 17$ & 0.004 \\
\hline Diastolic blood pressure (mm Hg) & $80 \pm 11$ & $79 \pm 11$ & $83 \pm 9$ & $83 \pm 11$ & $88 \pm 11$ & 0.14 \\
\hline Pulse pressure (mm Hg) & $45 \pm 10$ & $44 \pm 10$ & $48 \pm 9$ & $50 \pm 10$ & $50 \pm 10$ & 0.01 \\
\hline Total cholesterol (mg/dL) & $196 \pm 39$ & $193 \pm 38$ & $202 \pm 34$ & $207 \pm 41$ & $200 \pm 42$ & 0.21 \\
\hline HDL (mg/dL) & $51 \pm 14$ & $53 \pm 14$ & $49 \pm 11$ & $48 \pm 13$ & $41 \pm 10$ & $<0.0001$ \\
\hline Triglycerides (mg/dL) & $124 \pm 84$ & $109 \pm 62$ & $131 \pm 92$ & $158 \pm 96$ & $212 \pm 176$ & $<0.0001$ \\
\hline Fasting glucose (mg/dL) & $92 \pm 16$ & $86 \pm 7$ & $105 \pm 4$ & $95 \pm 12$ & $137 \pm 42$ & $<0.0001$ \\
\hline 2-hour glucose (mg/dL) & $116 \pm 33$ & $104 \pm 19$ & $106 \pm 19$ & $160 \pm 15$ & $211 \pm 40$ & $<0.0001$ \\
\hline Uric acid (mg/dL) & $4.9 \pm 1.3$ & $4.7 \pm 1.4$ & $5.1 \pm 1.2$ & $5.4 \pm 1.1$ & $5.2 \pm 1.4$ & $<0.0001$ \\
\hline hsCRP (mg/L) & $2.6 \pm 2.6$ & $2.4 \pm 2.4$ & $2.1 \pm 2.9$ & $3.5 \pm 2.8$ & $3.0 \pm 1.7$ & 0.003 \\
\hline Intima-media thickness (mm) & $0.71 \pm 0.15$ & $0.69 \pm 0.15$ & $0.75 \pm 0.14$ & $0.73 \pm 0.14$ & $0.87 \pm 0.15$ & 0.002 \\
\hline Insulin-stimulated glucose disposal & $8.7 \pm 4.3$ & $9.5 \pm 4.4$ & $8.9 \pm 3.9$ & $6.5 \pm 3.4$ & $5.4 \pm 2.8$ & $<0.0001$ \\
(mg/min $\times$ kg FFM) & & & & & & \\
\hline Steady-state plasma insulin levels & $86 \pm 33$ & $84 \pm 33$ & $92 \pm 32$ & $93 \pm 33$ & $78 \pm 24$ & 0.094 \\
during the clamp (mU/L) & & & & & & $<0.15$ \\
\hline Triglyceride/HDL-C ratio & $2.81 \pm 2.65$ & $2.37 \pm 1.89$ & $3.08 \pm 3.1$ & $3.63 \pm 2.86$ & $6.15 \pm 5.93$ & $<0.0001$ \\
\hline LAP index & $53.8 \pm 48.8$ & $45.2 \pm 38.1$ & $49.5 \pm 32.1$ & $79.6 \pm 59.1$ & $109.5 \pm 97.7$ & $<0.0001$ \\
\hline VAl & $1.93 \pm 1.83$ & $1.63 \pm 1.20$ & $1.93 \pm 1.82$ & $2.59 \pm 2.08$ & $4.28 \pm 4.61$ & $<0.0001$ \\
\hline TyG index & $9.17 \pm 0.59$ & $9.03 \pm 0.51$ & $9.37 \pm 0.53$ & $9.48 \pm 0.53$ & $10.1 \pm 0.73$ & $<0.0001$ \\
\hline
\end{tabular}

Data are means \pm SD. Triglyceride/HDL-C concentration ratio, LAP, VAl indexes, hsCRP, and triglyceride values were log transformed for statistical analysis, but values in the table represent back transformation to the original scale. Categorical variables were compared by $\chi^{2}$ test. Comparisons between the study groups were performed using a general linear model. $\mathrm{P}$ values refer to results after analyses with adjustment for age and gender.

${ }^{*} \mathrm{P}$ values refer to results after analyses with adjustment for gender.

BMI, body mass index; FFM, fat-free mass; HDL, high-density lipoprotein; HDL-C, high-density lipoprotein cholesterol; hsCRP, highsensitivity C-reactive protein; IFG, impaired fasting glucose; IGT, impaired glucose tolerance; LAP, lipid accumulation product; NGT, normal glucose tolerance; TyG, triglycerides $\times$ fasting glucose; VAI, visceral adiposity index.

atherosclerosis, defined as IMT $>0.9 \mathrm{~mm}$, and increased vascular stiffness, defined as pulse pressure $\geq 60 \mathrm{~mm} \mathrm{Hg}$. As shown in table 5, and online supplementary figures 2 and 3 , no differences were observed in the ability of the four surrogate indexes in detecting individuals with atherosclerosis as indicated by similar values of AUCs. By contrast, LAP index had the greatest ability to detect individuals with increased vascular stiffness as assessed by the higher value of the AUC (table 5).

Table 2 Pearson's correlation coefficient relating surrogate indexes of insulin resistance to insulin-stimulated glucose disposal $\left(\mathrm{M}_{\mathrm{FFM}}\right)$ across glucose tolerance categories

\begin{tabular}{lllllllllll}
\hline & $\begin{array}{l}\text { Whole } \\
\text { sample } \\
(\mathbf{n}=631)\end{array}$ & P value & $\begin{array}{l}\text { NGT } \\
(\mathbf{n}=446)\end{array}$ & P value & $\begin{array}{l}\text { IFG } \\
(\mathbf{n}=60)\end{array}$ & P value & $\begin{array}{l}\text { IGT } \\
(\mathbf{n}=95)\end{array}$ & $\begin{array}{l}\text { P value } \\
\text { diabetes }\end{array}$ & P value \\
\hline $\begin{array}{l}\text { Triglyceride/HDL-C } \\
\text { ratio }\end{array}$ & -0.411 & $<0.0001$ & -0.407 & $<0.0001$ & -0.292 & 0.01 & -0.109 & 0.14 & -0.314 & 0.09 \\
LAP index & -0.469 & $<0.0001$ & -0.442 & $<0.0001$ & -0.488 & $<0.0001$ & -0.252 & 0.007 & -0.472 & 0.008 \\
VAI & -0.392 & $<0.0001$ & -0.372 & $<0.0001$ & -0.316 & 0.007 & -0.129 & 0.10 & -0.327 & 0.07 \\
TyG index & -0.385 & $<0.0001$ & -0.374 & $<0.0001$ & -0.268 & 0.01 & -0.028 & 0.39 & -0.332 & 0.07 \\
\hline
\end{tabular}

HDL-C, high-density lipoprotein cholesterol; IFG, impaired fasting glucose; IGT, impaired glucose tolerance; LAP, lipid accumulation product; NGT, normal glucose tolerance; TyG, triglycerides $\times$ fasting glucose; VAl, visceral adiposity index. 


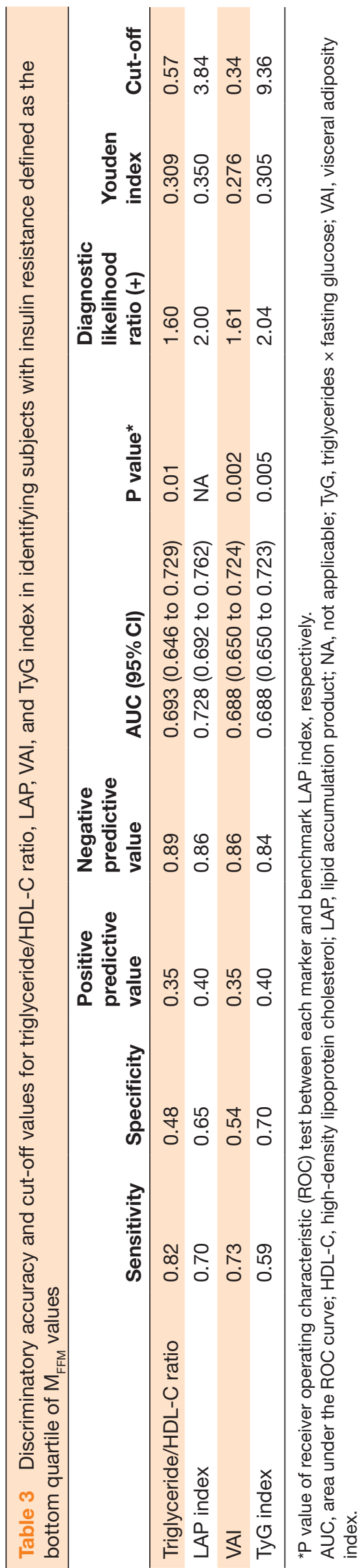

\section{DISCUSSION}

Compelling evidence suggests that insulin resistance, defined as decreased sensitivity of target organs to the metabolic actions of insulin, is a pathophysiological feature of the metabolic syndrome, and pre-diabetes, and heralds both type 2 diabetes and cardiovascular disease. ${ }^{1-11}$ The gold standard method for assessing insulin sensitivity is the euglycemic hyperinsulinemic clamp technique. ${ }^{12}$ Nonetheless, the glucose clamp method is technically demanding, invasive, costly, and time consuming, thus impeding its application in large epidemiological studies. Therefore, surrogate indexes based on inexpensive anthropometric and/or laboratory parameters collected in routine clinical practice have been developed to quantify insulin sensitivity. ${ }^{13-28}$ Given the extensive use of surrogate indexes of insulin sensitivity in epidemiological studies, it is important to compare their relationships with insulin sensitivity assessed by the euglycemic hyperinsulinemic clamp, and establish which surrogate index is the most predictive of insulin resistance. To address this issue, we compare four surrogate indexes based on anthropometric and/or laboratory parameters, that is, TG/HDL-C ratio, VAI, LAP and TyG index, with insulin sensitivity directly measured by the euglycemic hyperinsulinemic clamp method in 631 individuals having different degrees of glucose tolerance. We found that worsening of glycemic homeostasis from NGT status to type 2 diabetes was associated with a graded increase in the values of the four surrogate indexes of insulin resistance. However, LAP index was a better correlate of insulin sensitivity than VAI, TyG, and TG/HDL-C ratio indexes, and was the only index showing significant association with the insulin sensitivity in individuals with IGT or type 2 diabetes. Accordingly, based on the ROC analysis, LAP was the best index in discriminating individuals with insulin resistance because it was the only measure with an AUC value higher than the reference threshold of 0.7 , suggesting that LAP index offers more clinical information than that achieved by using VAI, TyG, and TG/HDL-C ratio indexes. To the best of our knowledge, this is the first systematic study that simultaneously compared VAI, LAP, TyG, and TG/ HDL-C ratio indexes with the euglycemic hyperinsulinemic clamp method in a large data set to determine which index is most reliable. Indeed, previous studies have examined the relationship between these four indexes and insulin resistance ${ }^{18-28}$; however, these studies differ from the present analysis for some important aspects. First, in most studies, ${ }^{18-25}$ insulin resistance was assessed by the HOMA-IR index, which is another surrogate index of insulin resistance derived from fasting glucose and insulin levels, and is thought to primarily reflect hepatic insulin resistance. By contrast, the euglycemic hyperinsulinemic clamp method was performed under steadystate insulin levels capable to suppress hepatic glucose production allowing direct assessment of glucose disposal predominantly into skeletal muscle. Second, a few studies have addressed the relative values of multiple insulin 
Table 4 Pearson's correlation coefficient relating surrogate indexes of insulin resistance to cardiometabolic risk factors and subclinical vascular damage

\begin{tabular}{lllllllll}
\hline & $\begin{array}{l}\text { Triglyceride/ } \\
\text { HDL-C ratio }\end{array}$ & P value & $\begin{array}{l}\text { LAP } \\
\text { index }\end{array}$ & P value & VAl & P value & $\begin{array}{l}\text { TyG } \\
\text { index }\end{array}$ & P value \\
\hline Systolic blood pressure $(\mathrm{mm} \mathrm{Hg})$ & 0.296 & $<0.0001$ & 0.421 & $<0.0001$ & 0.268 & $<0.0001$ & 0.358 & $<0.0001$ \\
Diastolic blood pressure $(\mathrm{mm} \mathrm{Hg})$ & 0.293 & $<0.0001$ & 0.406 & $<0.0001$ & 0.260 & $<0.0001$ & 0.338 & $<0.0001$ \\
Pulse pressure (mm Hg) & 0.152 & $<0.0001$ & 0.229 & $<0.0001$ & 0.145 & $<0.0001$ & 0.203 & $<0.0001$ \\
Uric acid (mg/dL) & 0.437 & $<0.0001$ & 0.449 & $<0.0001$ & 0.346 & $<0.0001$ & 0.383 & $<0.0001$ \\
hsCRP (mg/L) & 0.267 & $<0.0001$ & 0.443 & $<0.0001$ & 0.314 & $<0.0001$ & 0.234 & $<0.0001$ \\
Intima-media thickness $(\mathrm{mm})$ & 0.233 & $<0.0001$ & 0.247 & $<0.0001$ & 0.212 & $<0.0001$ & 0.276 & $<0.0001$ \\
\hline
\end{tabular}

HDL-C, high-density lipoprotein cholesterol; hsCRP, high-sensitivity C-reactive protein; LAP, lipid accumulation product; TyG, triglycerides $\times$ fasting glucose; VAl, visceral adiposity index.

sensitivity indexes in the same study group. ${ }^{18-28}$ Additionally, in the three studies that have compared the TyG, and TG/HDL-C ratio indexes with direct measure of insulin sensitivity by the euglycemic hyperinsulinemic clamp method, ${ }^{26}$ the SSPG concentration during the insulin suppression test $^{27}$ or a 3-hour hyperglycemic clamp test, ${ }^{28}$ the authors analyzed the relationships between the surrogate indexes with insulin sensitivity directly assessed in the whole group including indistinctly individuals with NGT, IFG and type 2 diabetes, thus lacking to determine whether the surrogate indexes are equally good correlates in individuals with different conditions of glucose tolerance. This difference is clinically meaningful since we have observed that among the examined indexes, only LAP index exhibited a significant association with insulin sensitivity in individuals with IGT or type 2 diabetes.

All the four indexes are highly significantly associated with cardiovascular risk factors, and subclinical vascular damage measured as IMT. The strength by which the four surrogate indexes correlate with cardiovascular risk factors was similar. Moreover, based on the ROC analysis, LAP,VAI, TyG, and TG/HDL-C ratio indexes are equally fair discriminators of vascular atherosclerosis defined as IMT $>0.9 \mathrm{~mm}$ because all of them pass the reference threshold of 0.7. By contrast, the ROC analysis indicates that the four indexes are poor discriminators of increased vascular stiffness defined as pulse pressure $>60 \mathrm{~mm} \mathrm{Hg}$ as none of them showed an AUC value higher than 0.7 (table 5).

The present study has some strengths. The main strength is that surrogate indexes were evaluated versus the euglycemic hyperinsulinemic clamp gold standard test in a large sample of subjects of both genders with various degrees of glucose tolerance. All tests including anthropometric measures, OGTT, and euglycemic hyperinsulinemic clamp studies were collected at the same time by a trained staff after a standardized training. Nonetheless, the current study has some limitations that should be considered. First, glucose tolerance by OGTT was only measured once. According to the ADA recommendations, ${ }^{35}$ one positive test is not sufficient to establish the degree of glucose

Table 5 Discriminatory accuracy and cut-off values for triglyceride/HDL-C ratio, LAP, VAI, and TyG index in identifying individuals with subclinical vascular damage

\begin{tabular}{|c|c|c|c|c|c|c|c|c|c|}
\hline & Sensitivity & Specificity & $\begin{array}{l}\text { Positive } \\
\text { predictive } \\
\text { value }\end{array}$ & $\begin{array}{l}\text { Negative } \\
\text { predictive } \\
\text { value }\end{array}$ & AUC $(95 \% \mathrm{Cl})$ & P value* & $\begin{array}{l}\text { Diagnostic } \\
\text { likelihood } \\
\text { ratio (+) }\end{array}$ & $\begin{array}{l}\text { Youden } \\
\text { index }\end{array}$ & Cut-off \\
\hline \multicolumn{10}{|c|}{ Vascular atherosclerosis defined as IMT $>0.9 \mathrm{~mm}$} \\
\hline $\begin{array}{l}\text { Triglyceride/ } \\
\text { HDL-C ratio }\end{array}$ & 0.950 & 0.412 & 0.134 & 0.989 & 0.701 (0.656 to 0.742$)$ & 0.15 & 1.62 & 0.362 & 0.499 \\
\hline LAP index & 0.900 & 0.494 & 0.146 & 0.981 & 0.742 (0.699 to 0.782$)$ & NA & 1.78 & 0.394 & 3.623 \\
\hline TyG index & 0.825 & 0.592 & 0.163 & 0.972 & 0.739 (0.696 to 0.779$)$ & 0.91 & 2.02 & 0.417 & 9.192 \\
\hline \multicolumn{10}{|c|}{ Increased vascular stiffness defined as pulse pressure $>60 \mathrm{~mm} \mathrm{Hg}$} \\
\hline $\begin{array}{l}\text { Triglyceride/ } \\
\text { HDL-C ratio }\end{array}$ & 0.662 & 0.473 & 0.169 & 0.897 & 0.551 (0.510 to 0.590$)$ & 0.001 & 1.26 & 0.136 & 0.659 \\
\hline LAP index & 0.721 & 0.500 & 0.189 & 0.917 & 0.614 (0.574 to 0.652$)$ & NA & 1.44 & 0.221 & 3.664 \\
\hline
\end{tabular}

${ }^{*} \mathrm{P}$ value of receiver operating characteristic $(\mathrm{ROC})$ test between each marker and benchmark LAP index, respectively.

$A \cup C$, area under the ROC curve; HDL-C, high-density lipoprotein cholesterol; IMT, intima-media thickness; LAP, lipid accumulation product; NA, not applicable; TyG, triglycerides $\times$ fasting glucose; VAI, visceral adiposity index. 
tolerance, which should be defined on the basis of at least two separate measurements, and therefore, some participants might have been misclassified. Second, endogenous glucose production during clamp studies has not been directly assessed by tracer methods, therefore we cannot firmly exclude that a residual hepatic glucose production occurs in some participants. Furthermore, the study was carried on a European population, and needs to be replicated in other ethnic groups before its widespread applicability. Additionally, the cross-sectional design of the study lacking prospective data impedes any assumption about cause and temporal relationships. Additionally, arterial stiffness was not directly assessed by measuring pulse wave velocity, but pulse pressure was used as proxy of arterial stiffness. And finally, residual confounding by unmeasured factors cannot be excluded.

In conclusion, the findings of the present study suggest that LAP index is a more reliable discriminator of insulin resistance in clinical settings as compared with VAI, TyG, and TG/HDL-C ratio indexes. The better ability of LAP index to identify individuals with insulin resistance compared with the other surrogate measures may, of clinical relevance, be due to the fact that it is based on inexpensive measurements of waist circumference and TGs easily available in clinical practice, which are also accessible in high-risk groups of undeveloped countries for estimating insulin resistance.

Contributors TVF researched the data, edited the manuscript and contributed to analyze the data and to write the manuscript. MAM and ES researched the data and contributed to the discussion. FA researched the data and reviewed the manuscript. GS conceived the study, analyzed the data and wrote the manuscript.

Funding The authors have not declared a specific grant for this research from any funding agency in the public, commercial or not-for-profit sectors.

Competing interests None declared.

Patient consent for publication Not required.

Ethics approval The study was approved by the hospital ethical committee (Comitato Etico Azienda 0spedaliera 'Mater Domini') and written informed consent was obtained from all participants in accordance with the principles of the Declaration of Helsinki.

Provenance and peer review Not commissioned; externally peer reviewed.

Data availability statement Data are available upon reasonable request.

Open access This is an open access article distributed in accordance with the Creative Commons Attribution Non Commercial (CC BY-NC 4.0) license, which permits others to distribute, remix, adapt, build upon this work non-commercially, and license their derivative works on different terms, provided the original work is properly cited, appropriate credit is given, any changes made indicated, and the use is non-commercial. See: http://creativecommons.org/licenses/by-nc/4.0/.

ORCID iD

Teresa Vanessa Fiorentino http://orcid.org/0000-0001-9485-8793

\section{REFERENCES}

1 Nathan DM, Davidson MB, DeFronzo RA, et al. Impaired fasting glucose and impaired glucose tolerance: implications for care. Diabetes Care 2007;30:753-9.

2 Marini MA, Succurro E, Castaldo E, et al. Cardiometabolic risk profiles and carotid atherosclerosis in individuals with prediabetes identified by fasting glucose, postchallenge glucose, and hemoglobin A1c criteria. Diabetes Care 2012;35:1144-9.

3 Fiorentino TV, Marini MA, Andreozzi F, et al. One-hour postload hyperglycemia is a stronger predictor of type 2 diabetes than impaired fasting glucose. J Clin Endocrinol Metab 2015;100:3744-51.

4 Reaven GM, Lecture B. Role of insulin resistance in human disease. Diabetes 1988;37:1595-607.

5 Sesti G, Capaldo B, Cavallo Perin P, et al. Correspondence between the International diabetes Federation criteria for metabolic syndrome and insulin resistance in a cohort of Italian nondiabetic Caucasians: the GISIR database. Diabetes Care 2007;30:e33.

6 Cheal KL, Abbasi F, Lamendola C, et al. Relationship to insulin resistance of the adult treatment panel III diagnostic criteria for identification of the metabolic syndrome. Diabetes 2004;53:1195-200.

7 Warram JHet al. Slow glucose removal rate and hyperinsulinemia precede the development of type II diabetes in the offspring of diabetic parents. Ann Intern Med 1990;113:909-15.

8 Lillioja S, Mott DM, Spraul M, et al. Insulin resistance and insulin secretory dysfunction as precursors of non-insulin-dependent diabetes mellitus: prospective studies of Pima Indians. N Engl J Med 1993;329:1988-92.

9 Howard G, O'Leary DH, Zaccaro D, et al. Insulin sensitivity and atherosclerosis. Circulation 1996;93:1809-17.

10 Yip J, Facchini FS, Reaven GM. Resistance to insulin-mediated glucose disposal as a predictor of cardiovascular disease. J Clin Endocrinol Metab 1998;83:2773-6.

11 Bonora E, Kiechl S, Willeit J, et al. Insulin resistance as estimated by homeostasis model assessment predicts incident symptomatic cardiovascular disease in Caucasian subjects from the general population: the Bruneck study. Diabetes Care 2007;30:318-24.

12 DeFronzo RA, Tobin JD, Andres R. Glucose clamp technique: a method for quantifying insulin secretion and resistance. Am J Physiol 1979;237:E214-23.

13 Lorenzo C, Haffner SM, Stančáková A, et al. Relation of direct and surrogate measures of insulin resistance to cardiovascular risk factors in nondiabetic Finnish offspring of type 2 diabetic individuals. J Clin Endocrinol Metab 2010;95:5082-90.

14 Otten J, Ahrén B, Olsson T. Surrogate measures of insulin sensitivity vs the hyperinsulinaemic-euglycaemic clamp: a meta-analysis. Diabetologia 2014;57:1781-8.

15 Abbasi F, Silvers A, Viren J, et al. Relationship between several surrogate estimates of insulin resistance and a direct measure of insulin-mediated glucose disposal: comparison of fasting versus post-glucose load measurements. Diabetes Res Clin Pract 2018;136:108-15.

16 Amato MC, Giordano C, Galia M, et al. Visceral adiposity index: a reliable indicator of visceral fat function associated with cardiometabolic risk. Diabetes Care 2010;33:920-2.

17 Kahn HS. The "lipid accumulation product" performs better than the body mass index for recognizing cardiovascular risk: a populationbased comparison. BMC Cardiovasc Disord 2005;5:26.

18 Xia C, Li R, Zhang S, et al. Lipid accumulation product is a powerful index for recognizing insulin resistance in non-diabetic individuals. Eur J Clin Nutr 2012;66:1035-8.

19 Sambataro M, Perseghin G, Lattuada G, et al. Lipid accumulation in overweight type 2 diabetic subjects: relationships with insulin sensitivity and adipokines. Acta Diabetol 2013;50:301-7.

20 Irace C, Carallo C, Scavelli FB, et al. Markers of insulin resistance and carotid atherosclerosis. A comparison of the homeostasis model assessment and triglyceride glucose index. Int J Clin Pract 2013;67:665-72

21 Du T, Yuan G, Zhang M, et al. Clinical usefulness of lipid ratios, visceral adiposity indicators, and the triglycerides and glucose index as risk markers of insulin resistance. Cardiovasc Diabetol 2014;13:146.

22 Mazidi M, Kengne A-P, Katsiki N, et al. Lipid accumulation product and triglycerides/glucose index are useful predictors of insulin resistance. J Diabetes Complications 2018;32:266-70.

23 LK E, Wu S, Chou HH, et al. Triglyceride glucose-body mass index is a simple and clinically useful surrogate marker for insulin resistance in nondiabetic individuals. PLoS One 2016;11:e0149731.

24 Lee SB, Ahn CW, Lee BK, et al. Association between triglyceride glucose index and arterial stiffness in Korean adults. Cardiovasc Diabetol 2018;17:41.

25 Ahn N, Baumeister SE, Amann U, et al. Visceral adiposity index (VAI), lipid accumulation product (LAP), and product of triglycerides and glucose (TyG) to discriminate prediabetes and diabetes. Sci Rep 2019;9:9693.

26 Guerrero-Romero F, Simental-Mendía LE, González-Ortiz M, et al. The product of triglycerides and glucose, a simple measure of insulin sensitivity. Comparison with the euglycemic-hyperinsulinemic clamp. J Clin Endocrinol Metab 2010;95:3347-51. 
27 Abbasi F, Reaven GM. Comparison of two methods using plasma triglyceride concentration as a surrogate estimate of insulin action in nondiabetic subjects: triglycerides $\times$ glucose versus triglyceride/ high-density lipoprotein cholesterol. Metabolism 2011;60:1673-6.

28 Vasques ACJ, Novaes FS, de Oliveira MdaS, et al. TyG index performs better than HOMA in a Brazilian population: a hyperglycemic clamp validated study. Diabetes Res Clin Pract 2011;93:e98-100.

29 O'Leary DH, Polak JF, Kronmal RA, et al. Carotid artery intima and media thickness as a risk factor for myocardial infarction and stroke in older adults. N Engl J Med 1999;340:14-22.

30 Cardellini M, Marini MA, Frontoni S, et al. Carotid artery intima-media thickness is associated with insulin-mediated glucose disposal in nondiabetic normotensive offspring of type 2 diabetic patients. Am J Physiol Endocrinol Metab 2007;292:E347-52.

31 Fiorentino TV, Succurro E, Arturi F, et al. Serum lgG2 levels are specifically associated with whole-body insulin-mediated glucose disposal in non-diabetic offspring of type 2 diabetic individuals: a cross-sectional study. Sci Rep 2018;8:13616.
32 Williams B, Mancia G, Spiering W, et al. ESC/ESH guidelines for the management of arterial hypertension. Eur Heart $J$ 2018;2018:3021-104.

33 Marini MA, Frontoni S, Mineo D, et al. The Arg ${ }^{972}$ Variant in Insulin Receptor Substrate-1 Is Associated with an Atherogenic Profile in Offspring of Type 2 Diabetic Patients. J Clin Endocrinol Metab 2003;88:3368-71.

34 Succurro E, Marini MA, Arturi F, et al. Elevated one-hour post-load plasma glucose levels identifies subjects with normal glucose tolerance but early carotid atherosclerosis. Atherosclerosis 2009;207:245-9.

35 American Diabetes Association. 2. Classification and Diagnosis of Diabetes: Standards of Medical Care in Diabetes - 2019. Diabetes Care 2019;42:S13-28.

36 DeLong ER, DeLong DM, Clarke-Pearson DL. Comparing the areas under two or more correlated receiver operating characteristic curves: a nonparametric approach. Biometrics 1988;44:837-45. 